\title{
Treatment of acromegaly increases BMD but reduces trabecular bone score: a longitudinal study
}

\author{
Kristin Godang ${ }^{1}$, Nicoleta Cristina Olarescu ${ }^{1}$, Jens Bollerslev ${ }^{1,2}$ and Ansgar Heck ${ }^{1,2}$ \\ ${ }^{1}$ Section of Specialized Endocrinology, Department of Endocrinology, Oslo University Hospital \\ Rikshospitalet, Oslo, Norway and 'Faculty of Medicine, University of Oslo, Oslo, Norway
}

Correspondence should be addressed to $\mathrm{K}$ Godang

Email

kgodang@ous-hf.no

\begin{abstract}
Context: Bone turnover is increased in acromegaly. Despite normalization of bone turnover after treatment, the risk for vertebral fractures remains increased. Gonadal status, but not BMD, is correlated with vertebral fractures. Trabecular bone score (TBS) is related to bone microarchitecture.

Objective: The aim of this study is to assess the longitudinal change in TBS and BMD following treatment for acromegaly.

Design, Setting, Patients, Interventions, and Main outcome measures: This longitudinal study included 48 patients with acromegaly between 2005 and 2015. BMD, TBS, and markers for bone turnover (P1NP and CTX-1) were measured at baseline and following treatment.

Results: Following treatment, the mean TBS decreased by $3.0( \pm 7.0) \%$, whereas the BMD at the lumbar spine (LS) increased by $3.2( \pm 4.9) \%$ (both $P<0.01)$. The changes in BMD LS and TBS were not correlated $(P=0.87)$.

The TBS change was found to be $-4.5 \%( \pm 6.7 ; P=0.003)$ in men and $-0.3 \%( \pm 6.8 ; P=0.85)$ in women $(P=0.063$ for interaction men vs women). The mean BMD LS increased in men $+4.2 \mathrm{~g} / \mathrm{cm}^{2}( \pm 4.3 ; P<0.001)$, but not in women $+1.5 \mathrm{~g} /$ $\mathrm{cm}^{2}$ ( $\left.\pm 5.6 ; P=0.36\right)$; $(P=0.073$ for interaction). BMD increased in the ultradistal radius and total body (both $P<0.01)$. The increase in BMD LS was associated with a decrease in P1NP and CTX-1 $(P<0.001)$ and with lower P1NP and CTX-1 at the follow-up $(P<0.02)$.

Conclusion: Treatment of acromegaly affects TBS and BMD at LS in different manners. The reduction of bone turnover markers predicts the increase in BMD but not the decrease in TBS. The DXA changes were more pronounced in men. Alterations in trabecular bone architecture may explain the persistent fracture risk despite the increase in BMD after disease control.

European Journal of Endocrinology

(2016) 175, 155-164
\end{abstract}

\section{Introduction}

Acromegaly is a rare and insidious disease characterized by overproduction of growth hormone (GH) almost exclusively caused by a pituitary adenoma and consequently increased insulin-like growth factor 1 (IGF-1) (1). GH and IGF-1 are important regulators of bone growth modeling and remodeling (2). In patients with acromegaly, active disease is associated with increased bone turnover (3), and this condition is considered as one of the causes of secondary osteoporosis that can lead to
() 2016 European Society of Endocrinology Printed in Great Britain increased fracture risk and therefore increased morbidity and mortality (1). Low bone mineral density (BMD) is an accepted surrogate marker for fracture risk (4). Evaluating the predisposing factors for the increased fracture risk in patients with acromegaly is important when making personalized follow-up recommendations to prevent or treat low-energy fractures in these patients.

Data on BMD in acromegaly are conflicting; however, the effects of non-physiological secretion of GH and IGF-1 
appear to affect the bone quality negatively $(5,6)$. Despite normalization of bone turnover following treatment of acromegaly, the risk for vertebral fractures is still increased $(5,7)$. One might assume that trabecular and cortical bones are differently affected by GH disorders, due to various sensitivity to the GH excess in trabecular and cortical bones. Previous studies have revealed a differential effect of GH excess on the axial (70\% trabecular bone) and appendicular skeleton (90\% cortical bone) (8) and have suggested that the bone density might be inconsistent in relation to the bone area. As BMD reflects only bone quantity, and bone quality has been shown to be altered in acromegaly $(9,10)$, the fracture risk may be underestimated by BMD (5). Biochemical markers of bone turnover can provide further reliable information on the changes in the bone remodeling, also in a high turnover state as acromegaly (11). Accordingly, we have previously demonstrated reduced trabecular biomechanical competence in patients with active acromegaly (12), indicating that patients with acromegaly were predisposed to develop vertebral fractures independently of BMD. Therefore, it is important to explore non-invasive methods to assess bone microarchitecture and quality rather than just BMD in acromegaly (9).

As BMD alone only provides a measure on bone density, but does not assess the structural characteristics of the bone, a new and non-invasive technology, trabecular bone score (TBS), has been developed (13).

The TBS score gives an evaluation of bone microarchitecture based on the DXA image of the lumbar spine (LS). TBS is an analytical tool that performs graylevel texture measurements on DXA image of the LS and thereby captures information relating to trabecular microarchitecture $(14,15)$. Previous studies have demonstrated that TBS correlates with three-dimensional bone microarchitecture parameters, such as connectivity density and trabecular number, and negatively with trabecular separation $(16,17)$.

The external and internal architectures of bones are important factors for the distribution and transmission of loads (18). A combination of structural and densitometric indices might potentially provide a more accurate assessment of bone quality (19). TBS can be of importance where DXA results are in conflict with the clinical presentation. One reason for the discrepancy may be the presence of factors affecting not only bone density but also bone strength, such as bone microarchitecture, macro-geometry of the cortical bone, and bone turnover. TBS might provide information beyond and independently of BMD.
Disease control in acromegaly does not seem to reverse the increased fracture risk (7). Increased knowledge of the quantitative and qualitative processes in the skeleton of patients with acromegaly during treatment is necessary in order to develop strategies for prevention and treatment of the increased fracture risk.

Therefore, the primary aim of this study is to assess the longitudinal change of vertebral cancellous bone microarchitecture as assessed by TBS and site-matched BMD before and following treatment for acromegaly. The secondary aims are to study the BMD change in other compartments assessed by DXA and thereby enhance the understanding with correlations to biochemical markers of disease activity and bone turnover.

\section{Subjects and methods}

\section{Population, baseline characteristics, and treatment}

A total of 48 treatment-naïve patients with active acromegaly diagnosed at the Oslo University Hospital between 2005 and 2015 were included in the study. The diagnosis was based on clinical symptoms and confirmed by an elevated, age-adjusted IGF-1 level and failure to suppress GH by an oral glucose tolerance test. The inclusion criteria were (1) availability of a DXA scan at baseline (all patients, $n=48$ ) and (2) either after primary somatostatin analogue (SA) treatment at 6-12 months (SA visit) $(n=17)$ and/or after transsphenoidal pituitary surgery at 12 months ( 1 yrPO visit) $(n=38)$. Of the total number of patients, seven had DXA available both at SA and 1 yrPO visit. Patients were defined as hypogonadal during the study period if they did not receive any ethinyl estradiol or testosterone treatment and had serum estradiol, in female, and testosterone and/or free testosterone index (total testosterone/SHBG*10), in male, below the reference values. Accordingly, nine female and one male patient were hypogonadal; the rest of the patients were defined as eugonadal.

Blood samples were drawn in the morning after an overnight fast, and serum was collected and stored at $-80^{\circ} \mathrm{C}$ until analyzed.

\section{Disease activity and biochemical assays}

Plasma GH and IGF-1 were measured by immunoassay (IMMULITE 2000; Siemens Healthcare) (20). Sex steroid and gonadotropin analyses were performed by an accredited laboratory (Department of Medical Biochemistry, Oslo University Hospital Rikshospitalet, 
Norway) according to standard methods. Crosslaps, C-terminal telopeptides of type I collagen (CTX-1) was analyzed as a bone resorption marker and propeptide of type I procollagen (P1NP) as a bone formation marker. The bone markers were analyzed consecutively in duplicates in one run in order to minimize analytical variability. Serum CTX-1 was determined by an enzymelinked immunosorbent assay (ELISA; Immunodiagnostic Systems Nordic, Denmark). Serum P1NP (UniQ P1NP; Orion Diagnostica, Espoo, Finland) and 25-hydroxyvitamin D $(25(\mathrm{OH})$ D) (DiaSorin, Stillwater, MN, USA) were quantified by radioimmunoassay (RIA). According to the manufacturers, the reference values for P1NP and CTX-1 in adults are $<85 \mu \mathrm{g} / \mathrm{L}$ and $<0.291 \mu \mathrm{g} / \mathrm{L}$ respectively. Intra- and inter-assay coefficients of variation were $<10 \%$ for all assays. Serum for analyses of CTX-1, P1NP, and 25-hydroxyvitamin D was available in 46 patients at baseline, 14 patients at SA visit, and 33 patients at 1-year $\mathrm{PO}$ visit.

\section{BMD measurements}

BMD was determined by DXA at the lumbar spine (LS), anterior-posterior projection (BMD LS; L1-L4), bilateral proximal femur, total hip, and femoral neck; in the nondominant forearm, ultradistal radius, and proximal third of radius (proximal radius); and total body. A narrow fan beam GE Healthcare Lunar Prodigy densitometer was used and all the scans were analyzed using software version 14.10 (GE Healthcare Lunar Corp). No hardware changes were made during the study period. BMD LS was calculated as the mean value of the single vertebrae in each patient, after vertebrae with severe compression fractures were excluded. Only scans with at least two nonfractured vertebras were considered. All the analyses and the exclusion of compressed vertebra(e) were performed by an ISCD (The International Society for Clinical Densitometry) Certified Clinical Densitometrist (KG).

Absolute BMD values $\left(\mathrm{g} / \mathrm{cm}^{2}\right)$ and BMD Z-scores were estimated by comparison to the reference population incorporated in the software suitable for clinical use in the Norwegian population (21). Calibration of the DXA machine was performed daily using a calibration block consisting of tissue equivalent material with three bones, simulating chambers, as supplied by the manufacturer (22). The short- and long-term coefficients of variation for our densitometer were $0.8 \%$ and $1.4 \%$ respectively. The precision error at LS was about $1 \%$ (23), and according to the manufacturer, the determination of BMD total body has a precision error of $\sim 0.5 \%$.

\section{Assessment of TBS}

Spine TBS parameters were extracted from DXA L1-L4 images by using TBS iNsight software (v2.1.2.0; Medimaps Group, Geneva, Switzerland).

TBS is a textural index that evaluates pixel gray-level variations in the LS using two-dimensional (2D) projection images derived from the BMD DXA image. TBS represents an indirect index of trabecular microarchitecture (24). Based on experimental variograms of the projected DXA image, TBS has the potential to discern differences in 3D microarchitecture between 2D DXA measurements that are similar to each other $(14,24)$. BMD and TBS analyses were performed on the same vertebrae and same region of interest, so that the results can be directly compared. TBS at LS has a short-term in vivo precision of $1.12-1.9 \%$.

Although TBS was assessed for each vertebra, the reported TBS value represents the average of L1-L4 after exclusion of vertebra(e) with severe compression fracture.

The following range for TBS values in postmenopausal women has been proposed (24): TBS $\geq 1.350$ is considered normal; TBS between 1.200 and 1.350 is considered to be consistent with partially degraded microarchitecture; and TBS $\leq 1.200$ is considered as degraded microarchitecture. These cutoff points were established by a working group of TBS users from different countries, (25) by analogy with the three BMD categories, normal bone mass, osteopenia, and osteoporosis. A normal range for TBS in men has not yet been established (26).

\section{Ethics}

The study was approved by the Regional Ethics Committee for Southern Norway and was performed according to the Declaration of Helsinki. Written informed consent was obtained from all patients.

\section{Statistical analysis}

Descriptive data were presented as mean ( \pm s.D.) if normally distributed (Shapiro-Wilk test) and as median (25, 75 percentile) if not. Difference between time points (before and after treatment) for each parameter was assessed by paired sample $t$-test or Wilcoxon signed-rank test.

Interactions depending on gender and gonadal status were calculated by testing for significant differences in changes over time. Correlations between continuous variables were assessed by Pearson's and Spearman's correlation coefficient. Parametric correlation coefficients and $P$-values were indicated by lowercase letters $(\mathrm{r} ; \mathrm{p}$ ) 
and non-parametric correlation coefficients by uppercase letters (R; P). All statistical analyses were performed using SPSS, version 22 .

\section{Results}

\section{Patient characteristics}

Totally, 48 patients with acromegaly were diagnosed at a mean age of $47.7( \pm 14.6)$ years. Baseline characteristics, biochemical measurements, BMI, BMD, and Z-scores are presented in Table 1. Baseline demographics in the subcohorts (SA and 1 yrPO) were not significantly different.

In patients assessed 1 year postoperatively ( $1 \mathrm{yrPO})$, IGF-1 decreased from $109.4( \pm 43.1) \mathrm{nmol} / \mathrm{L}$ to 35.2 $( \pm 19.2) \mathrm{nmol} / \mathrm{L}$ and $\mathrm{GH}$ from $6.7(3.1 ; 15.8) \mu \mathrm{g} / \mathrm{L}$ to 0.9 (0.4; 2.7) $\mu \mathrm{g} / \mathrm{L}($ all $P<0.001)$.

In patients assessed after primary SA treatment, IGF-1 decreased from $125.7( \pm 31.3) \mathrm{nmol} / \mathrm{l}$ to 71.5 $( \pm 30.7) \mathrm{nmol} / \mathrm{L}$ and $\mathrm{GH}$ from $15.5(7.7 ; 34.5) \mu \mathrm{g} / \mathrm{L}$ to 2.7 $(1.3 ; 4.3) \mu \mathrm{g} / \mathrm{L}(P<0.001)$.

\section{Biochemical markers of bone turnover}

Median baseline P1NP and CTX-1 concentrations were elevated compared with reference values, $90(58 ; 127) \mu \mathrm{g} / \mathrm{L}$ and $0.97(0.69 ; 1.83) \mu \mathrm{g} / \mathrm{L}$ respectively. Mean $25(\mathrm{OH}) \mathrm{D}$ was $47.2( \pm 16.9) \mathrm{nmol} / \mathrm{L}$ at baseline.

Table 1 Baseline characteristics of study population. Data are presented as mean ( \pm S.D.) or median $(25,75$ percentile) if not stated otherwise.

\begin{tabular}{lc}
\hline$n^{\mathrm{a}}$ & $48(\mathrm{~F}: 17 ; \mathrm{M}: 31)$ \\
Age at diagnosis (years) & $47.7(14.6)$ \\
$\mathrm{BMI}\left(\mathrm{kg} / \mathrm{m}^{2}\right)$ & $28.5(4.1)$ \\
$\mathrm{IGF}-1(\mathrm{nmol} / \mathrm{L})$ & $115.6(88.2)$ \\
IGF-1/ULN & $2.9(1.8,3.6)$ \\
$\mathrm{GH}(\mu \mathrm{g} / \mathrm{L})$ & $8.7(5.0,20.8)$ \\
$25(\mathrm{OH}) \mathrm{D}(\mathrm{nmol} / \mathrm{L})$ & $47.2(16.9)$ \\
$\mathrm{P} 1 \mathrm{NP}(\mu \mathrm{g} / \mathrm{L})$ & $90(58,127)$ \\
$\mathrm{CTX}-1(\mu \mathrm{g} / \mathrm{L})$ & $0.97(0.69,1.83)$ \\
DXA & \\
TBS LS & $1.324(0.151)$ \\
LS; L1-L4 Z-score & $-0.15(1.50)$ \\
Total hip Z-score & $-0.11(1.07)$ \\
Femoral neck Z-score & $0.05(1.01)$ \\
Proximal radius Z-score* & $-0.60(1.29)$ \\
Ultradistal radius Z-score & $-0.09(1.63)$ \\
Total body Z-score & $-0.05(1.39)$ \\
\hline
\end{tabular}

F, female; M, male; 25(OH) D, 25-hydroxyvitamin D; BMl, body mass index; CTX-1, Crosslaps, C-terminal telopeptides of type I collagen; DXA,

dual-energy X-ray absorptiometry; IGF-1, insulin-like growth factor-l; P1NP, propeptide of type I procollagen; TBS, trabecular bone score. ${ }^{a} n=46$ for 25(OH) D, P1NP and CTX-1; *Significantly different from $0(P=0.003)$.
At 1 yrPO visit, median P1NP concentration decreased from $73(56 ; 136)$ to $27(17 ; 36) \mu g / L \quad(P<0.001)$ and CTX-1 concentration from $0.86(0.60 ; 1.79)$ to $0.36(0.22$; $0.54) \mu \mathrm{g} / \mathrm{L}(P<0.001)$ (Fig. 1).

At the SA visit, the bone turnover markers were also decreased compared with baseline: P1NP decreased from $113(91 ; 152) \mu \mathrm{g} / \mathrm{L}$ at baseline to $49(36 ; 77) \mu \mathrm{g} / \mathrm{L}$; CTX-1 decreased from $1.82(1.24 ; 2.31)$ to $0.77(0.37 ; 1.24)$ $(P<0.01)$ (Fig. 1).

The changes in IGF-1 and in biochemical markers of bone turnover were positively correlated (Fig. 2).
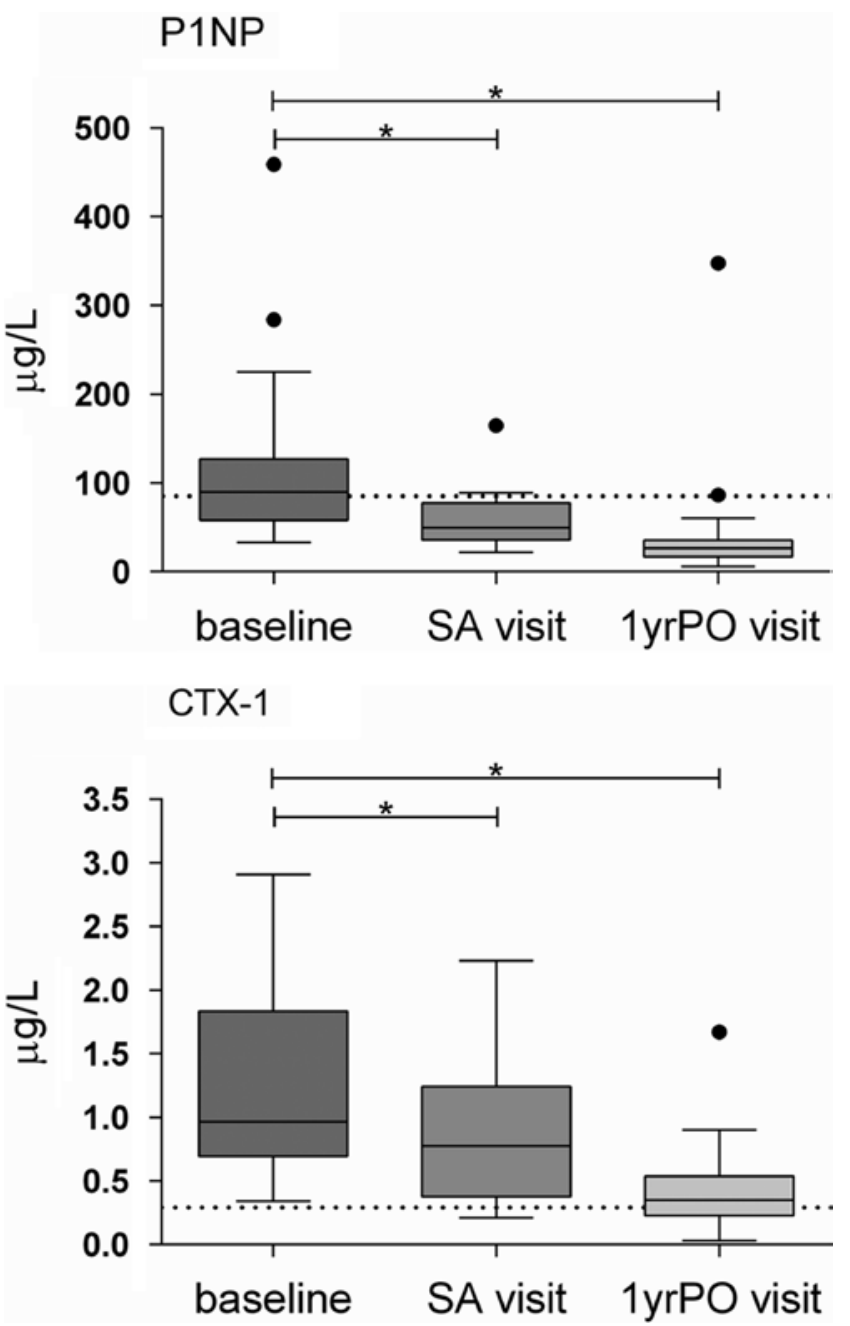

\section{Figure 1}

Bone markers decreased after treatment: P1NP and CTX-1 at baseline $(n=46)$, after $6-12$ months of primary treatment with SA $(n=14)$, and 1 year postoperatively $(n=33)$. The dashed horizontal line represents the upper limit of normal. Changes from baseline were significant $(P<0.05)$. Data are presented as mean \pm S.E.M. 

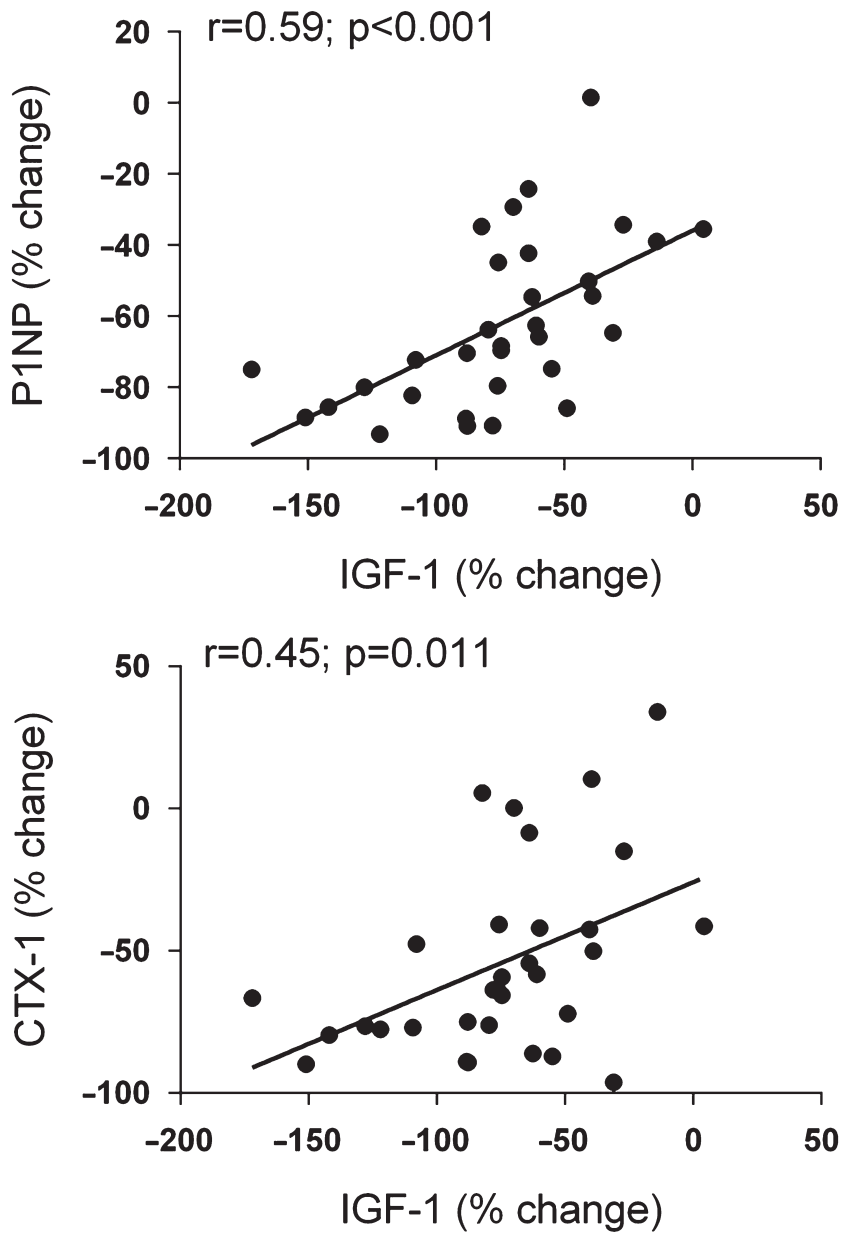

Figure 2

Change in IGF-1 and bone markers 1 year postoperatively: Correlation between changes from baseline: IGF-1 vs bone markers.

The correlation coefficients $(r)$ between the relative reduction of IGF-1 to bone markers (CTX-1 and P1NP) were $0.57(P=0.034)$ and $0.77(P=0.001)$ at SA visit and $0.57(P=0.001)$ and $0.49(P=0.005)$ at $1 \mathrm{yrPO}$ visit.

$25(\mathrm{OH}) \mathrm{D}$ did not change from baseline to any of the follow-up visits.

\section{Bone mineral measurements}

At baseline, the mean $Z$-scores did not differ from the zero, in LS, femoral neck, total hip, ultradistal radius, and total body, indicating no significant differences in patients to the age-matched reference population. In the proximal radius, the $Z$-score was -0.60 (95 \% CI: -0.98 to -0.22 ; $P=0.003$ ) (Table 1).
Table 2 Longitudinal measurements at baseline and 1-year postoperative visit of 38 (female: 14; male: 24 ) patients. Data are presented as mean ( \pm S.D.) or median $(25,75$ percentile) if not stated otherwise.

\begin{tabular}{|c|c|c|c|}
\hline & Baseline & 1-year PO & $P^{a}$ \\
\hline Age at diagnosis & $49.0(14.1)$ & NA & \\
\hline BMI $\left(\mathrm{kg} / \mathrm{m}^{2}\right)$ & $28.7(4.2)$ & $28.6(4.9)$ & \\
\hline \multicolumn{4}{|l|}{ DXA } \\
\hline TBS LS & $1.331(0.147)$ & $1.288(0.137)$ & $<0.001$ \\
\hline BMD LS; L1-L4 & $1.204(0.194)$ & $1.241(0.199)$ & $<0.001$ \\
\hline BMD total hip & $1.044(0.148)$ & $1.054(0.137)$ & .059 \\
\hline $\begin{array}{l}\text { BMD femoral } \\
\text { neck }\end{array}$ & $0.994(0.146)$ & $0.992(0.1$ & .896 \\
\hline $\begin{array}{l}\text { BMD proximal } \\
\text { radius }\end{array}$ & $0.880(0.138)$ & $0.894(0.138)$ & .099 \\
\hline $\begin{array}{l}\text { BMD ultradistal } \\
\text { radius }\end{array}$ & $0.478(0.092)$ & $0.514(0.097)$ & $<0.001$ \\
\hline BMD total body & $1.184(0.163)$ & $1.228(0.177)$ & $<0.001$ \\
\hline $25(\mathrm{OH}) \mathrm{D}(\mathrm{nmol})$ & 47 (19) & $51(21)$ & .360 \\
\hline P1NP ( $\mu \mathrm{g} / \mathrm{L})$ & $73(56,136)$ & $27(17,36)$ & $<0.001$ \\
\hline CTX-1 $(\mu \mathrm{g} / \mathrm{L})$ & $.86(0.60,1.79)$ & $0.36(0.22,0.54)$ & $<0.001$ \\
\hline IGF-1 (nmol/L) & $109.4(43.1)$ & $35.2(19.2)$ & $<0.001$ \\
\hline IGF-1/ULN & $2.8(1.6,3.5)$ & $0.8(0.6,1.3)$ & $<0.001$ \\
\hline $\mathrm{GH}(\mu \mathrm{g} / \mathrm{L})$ & $6.7(3.1,15.8)$ & $0.9(0.4,2.7)$ & $<0.001$ \\
\hline
\end{tabular}

BMD, bone mineral density; DXA, dual-energy X-ray absorptiometry; TBS, trabecular bone score.

aComparison between baseline and 1-year PO values.

At 1 yrPO visit, BMD LS, BMD ultradistal radius, and BMD total body had increased compared with baseline (BMD LS: $+3.2( \pm 4.9) \% ; P<0.001$ for all), and there was a trend toward an increase in BMD hip and BMD proximal radius $(P<0.1)$, whereas BMD femoral neck did not change significantly (Table 2 ).

At SA visit, only BMD LS increased significantly (Supplementary Table 1, see section on supplementary data given at the end of this article).

\section{Trabecular bone score}

Mean TBS at baseline $(n=48)$ was $1.324( \pm 0.151)$ and decreased significantly in the 1 yrPO cohort from 1.331 $( \pm 0.147)$ to $1.288( \pm 0.137)(P=0.007$; Table 2$)$. The changes in BMD LS and TBS were not correlated $(r=0.03 ; P=0.87)$. Baseline TBS was neither correlated with baseline BMD LS nor to the TBS change $(r=0.28, P=0.09 ; r=-0.04, P=0.79)$.

\section{Changes in TBS and BMD were different in women and men}

TBS decreased in men $(-0.065( \pm 0.094) P=0.003$ for change) but not in women $(-0.006( \pm 0.085) ; P=0.78$ for change) (Fig. 3) corresponding to a reduction of 4.5 
TBS

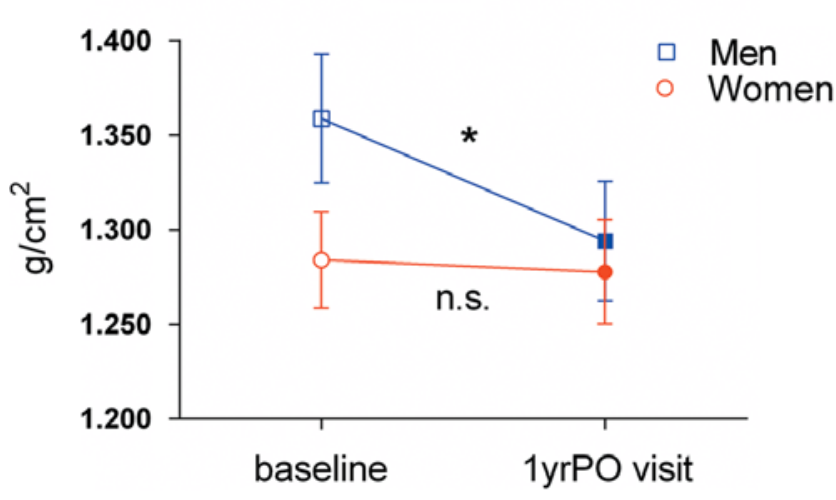

BMD

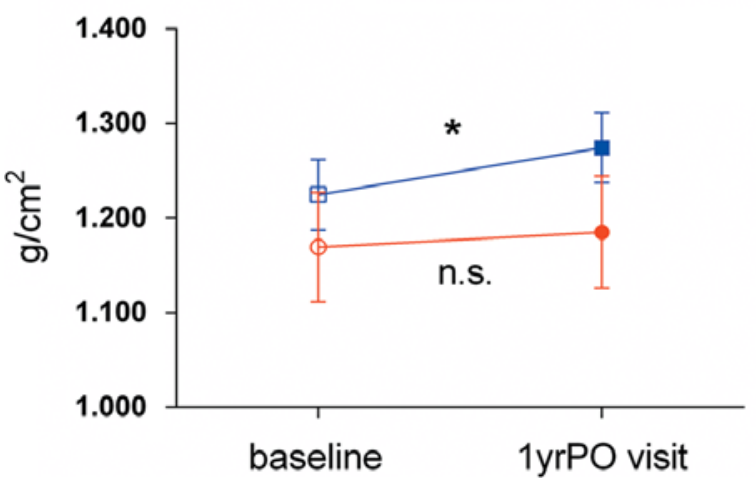

Figure 3

Gender difference in change in TBS and BMD lumbar spine. TBS decreased in men but not in women. There was a trend toward a significant difference between the changes in men vs women ( $P=0.063$ for interaction between gender groups). BMD increased in men but not in women ( $P=0.35$ for interaction). ( $\left.{ }^{*}<0.05\right)$. Data are presented as mean \pm S.E.M.

$( \pm 6.7) \%$ in men and $0.3( \pm 6.8) \%$ in women respectively. There was a trend toward a difference between the changes in men vs women $(P=0.063$ for interaction between gender groups).

The mean BMD LS increased to $+0.050 \mathrm{~g} / \mathrm{cm}^{2}( \pm 0.051$; $P \leq 0.001$ for change $)$ in men and $+0.016 \mathrm{~g} / \mathrm{cm}^{2}( \pm 0.061$; $P=0.35$ ) in women (Fig. 3) corresponding to an increase of $4.2( \pm 4.3) \%$ in men and $1.5( \pm 5.6) \%$ in women. There was a trend toward interaction depending on gender $(P=0.073)$.

If the cohort was analyzed stratified by gonadal status, TBS decreased in eugonadal subjects $(-0.05( \pm 0.08)$; $P=0.001)$, but not in hypogonadal subjects $(-0.02( \pm 0.14)$; $P=0.7$; interaction between groups: $P=0.36$, Fig. 4$)$. BMD LS increased significantly by $0.047( \pm 0.050) \mathrm{g} / \mathrm{cm}^{2}$ $(P<0.001$ for change) in eugonadal patients $(23$ males
TBS

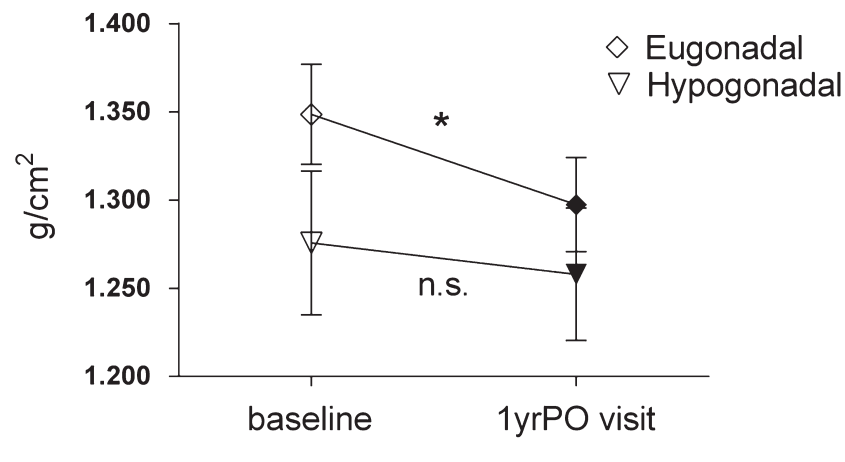

BMD

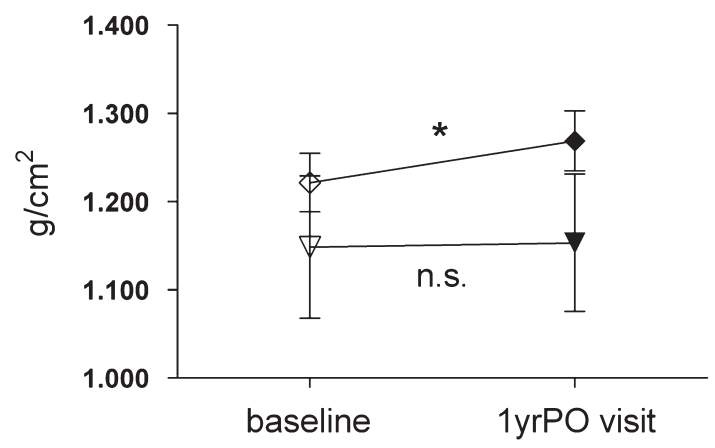

Figure 4

Change in TBS and BMD lumbar spine depends on gonadal status: TBS decreased in eugonadal but not in hypogonadal subjects ( $P=0.36$ for interaction between groups). BMD lumbar spine increased in eugonadal but not in hypogonadal subjects. The change was significantly different between eugonadal and hypogonadal subjects $(P=0.048$ for interaction). ( $\left.{ }^{*} P<0.05\right)$. Data are presented as mean \pm S.E.M.

and five females). In hypogonadal patients (one male and nine females), no significant change was detected $\left(-0.005( \pm 0.066) \mathrm{g} / \mathrm{cm}^{2} ; P\right.$ for change $=0.83$, Fig. 4$)$. There was a significant interaction depending on gonadal status $(P=0.048)$. Correspondingly, there was a greater increase in mean BMD total body in the eugonadal vs hypogonadal group (eugonadal: $+0.049( \pm 0.050) \mathrm{g} / \mathrm{cm}^{2}$; hypogonadal: $+0.017( \pm 0.027) \mathrm{g} / \mathrm{cm}^{2} ; P=0.036$ for interaction $)$.

\section{Other parameters associated with change in DXA parameters 1 year postoperatively}

Older patients tended to have a larger decrease in TBS ( $r=0.28 ; P=0.093)$, although there was no association between age and change in BMD LS $(r=0.09 ; P=0.6)$. High TBS at baseline was associated with larger decrease in TBS $(r=0.43 ; P=0.007)$. 
The change in TBS was not significantly associated with any of the tested variables (GH, IGF-1, BMI, bone markers, 25(OH) D, BMD LS or change in BMD LS, and bone markers or IGF-1).

The increase in BMD LS was associated with BMI at baseline $(r=0.52 ; P=0.001)$, low absolute values of bone turnover markers after treatment (P1NP: $r=-0.60$; $P<0.001$; CTX-1: $r=-0.39 ; P=0.024)$, and the \% decrease of bone turnover markers (P1NP: $r=0.47 ; P=0.007$; CTX-1: $r=0.49 ; P=0.005$ ).

\section{Discussion}

In the present longitudinal study of patients with active acromegaly undergoing treatment, a significant decrease in biochemical markers of disease activity was accompanied by an unexpected reduction in TBS for men and eugonadal patients, despite an increase in BMD in most compartments. The increase in BMD following treatment, as an estimate for bone mass, was expected due to the rapid fall in biochemical markers of bone turnover and thereby a closure of the bone remodeling space.

In this study, the rapid fall in IGF-1 was mirrored by a pronounced fall in CTX-1 as well as in P1NP seen already after 6 months of SA treatment, and even more pronounced at the $1 \mathrm{yrPO}$ visit. The biochemical markers reflect bone resorption (CTX-1) and formation (P1NP) and together they describe bone turnover $(11,27)$.

Bone turnover and bone area are increased in acromegaly, whereas bone mineral content seems to be unaltered, giving a relative decrease in BMD (11). The increase in bone area and thereby cortical thickness in the long bones might explain data from register studies, indicating a reduction in peripheral fractures in acromegaly (28). Bone anabolic agents as GH and IGF-1 are differently distributed in trabecular and cortical bone in patients with acromegaly (29), and interestingly, the ex vivo strength of trabecular bone was decreased in active disease (12), in accordance with a reduction in spinal volumetric bone mass by pQCT in uncontrolled patients with acromegaly (30).

Vertebral fractures are often silent and typically underestimated in frequency. Recently, several studies have shown a marked increase in vertebral fracture prevalence and incidence for both sexes in acromegaly $(5,31,32,33,34)$, related to the duration of disease activity and gonadal status among others. These observations underscore the complexity of a chronic and devastating disease as acromegaly, for bone as a target organ with fracture as the ultimate end point (2).
The only study so far assessing TBS in acromegaly was cross-sectional and showed decreased lumbar spine TBS in patients compared with controls, whereas BMD was not different between the two groups (35). In our longitudinal study, the mean TBS at baseline was partially degraded according to the definition by the manufacturer, whereas BMD $Z$-scores in most compartments did not differ from zero. Following treatment, however, an opposite effect on TBS and BMD was found. Bone remodeling is coupled in as well active acromegaly as following treatment (11), in contrast to for instance endogenous Cushing's syndrome, where bone resorption and formation are uncoupled in the active state, becoming coupled following treatment and remission (36). It is therefore to be expected that disease control in acromegaly will be followed by a decrease in bone turnover and a filling of the remodeling space followed by a relative increase in $\operatorname{BMD}(11,27)$, as seen in other endocrine disorders with coupled bone remodeling, following treatment $(37,38)$. The decrease in TBS following treatment in this study, despite the increase in BMD, reflects that the two measures describe different properties of bone, especially in other conditions than postmenopausal osteoporosis (4), but are in alignment with the observed high incidence and prevalence of vertebral fractures seen in acromegaly $(5,31,32,33,34)$.

GH and IGF-I are important anabolic hormones for bone but chronic excess, as long-lasting, active acromegaly seems to alter bone quality. Accordingly, increased cortical bone mass seems to be due to a direct effect of GH (periosteal apposition (11)) followed by an increase in bone size, but might have a detrimental effect on trabecular bone structure $(10,12,39)$. Prospective studies have demonstrated a high rate of incident vertebral fractures both in patients with active and controlled acromegaly despite a normal BMD $(7,34)$, suggesting that BMD has a limited usefulness in discriminating the patients who are posed to a high risk of fracture. Moreover, patients with acromegaly often have degenerative changes in the spine giving a false high BMD (5). Thus, bone strength depends not only on bone density but also on other dimensions of bones such as bone geometry, cortical thickness, and porosity, as well as trabecular bone morphology (4).

TBS is related to bone microarchitecture and predicts fracture risk in individuals with conditions related to reduced bone mass or bone quality (24). In this study, treatment leads to opposite changes in TBS and BMD in the whole cohort, but driven by men and eugonadal subjects. There was no change in TBS and BMD in women and hypogonadal subjects (most of them being women and just one hypogonadal man). Thus, the disease activity 
might have increased TBS more in the eugonadal patients and the decrease of disease activity leads to a more deleterious effect in these patients. Indeed, a previous study presented that the anabolic effect of chronic GH excess on trabecular bone, as assessed in spinal volumetric trabecular bone mass by single-energy quantitative computer tomography in a longitudinal retrospective study, was detectable only in eugonadal patients, whereas it was not observed in the presence of hypogonadism (30).

The increase in BMD occurring in men and a simultaneous decrease in TBS indicate that the increased bone density was unevenly distributed within the trabecular bone. Thereby, the increased bone density may not translate as effectively into increased bone stability as in situations where both BMD and TBS increase as in the treatment of postmenopausal osteoporosis (40).

Although an altered trabecular microstructure could explain the persistent increased risk for vertebral fractures even in controlled patients, our results should be interpreted with caution until a direct association has been proven and the potential errors for TBS in acromegaly accounted for. For example, as TBS is computed from DXA images, any image "noise" can influence the TBS evaluation and excessive soft tissue in the abdomen, overlying the region of interest, may reduce the TBS estimate. It is known that with treatment of acromegaly, although BMI remains unchanged, the patients do accumulate fat, predominantly in the central depots $(20,41)$, and this can interfere with TBS estimates and lead to a measurement of a false decrease in TBS.

The strength of this study is the relatively high number of well-characterized patients followed prospectively, although TBS was calculated retrospectively. Some technical limitations of TBS analyses should however be noted. Additional studies, both technical and clinical, should be performed to assess the advantages and limitations of the TBS in order to ensure that it is used appropriately, also in acromegaly, where the disease by itself, but also treatment will have major impact not only on bone tissue but also on surrounding tissue (20). In order to mitigate this problem in vivo, the TBS calculation is adjusted for BMI. The use of BMI, however, is limited as it cannot distinguish central abdominal weight accumulation, which would influence TBS derived from LS DXA, from adiposity at other sites. Of note, the adjustment in TBS for BMI is optimized when BMI is between 15 and $37 \mathrm{~kg} / \mathrm{m}^{2}$. Therefore, further longitudinal prospective studies are warranted taking into account prevalent and incident fractures so the TBS is proven to supply additional information about the trabecular microarchitecture. Dysfunctional bone remodeling can result in an increased fracture risk (10). As demonstrated previously, BMD does not reflect the increased fracture risk in acromegaly (5). Decreased TBS despite increased BMD might be a marker for dysfunctional bone remodeling. Thus, future studies should include both DXA parameters as TBS and BMD, bone turnover markers, and prospective vertebral and non-vertebral fracture assessment.

In conclusion, this study of patients with active acromegaly undergoing treatment appeared to have different effect on bone measures: (i) treatment of acromegaly affects TBS and BMD at LS in different and opposite manners - this effect is more pronounced in men and eugonadal subjects; (ii) the reduction of bone turnover markers determined the increase in BMD, following treatment; (iii) TBS decreased significantly and unexpectedly independent of BMD and bone turnover markers, following treatment; (iv) alterations in trabecular bone architecture related to the biomechanical properties might be reflected by TBS also in acromegaly and explain the increased fracture risk in these patients, independent of BMD.

\section{Supplementary data}

This is linked to the online version of the paper at http://dx.doi.org/10.1530/ EJE-16-0340.

Declaration of interest

$\mathrm{AH}$ has received speaker fees from Novartis, Ipsen, Novo Nordisk, and Pfizer and has participated in Novartis' Nordic Advisory Board. J B has received unrestricted research grants from Novartis, Pfizer, and Merck Norway AS. K G and N C O have nothing to disclose.

\section{Funding}

Ansgar Heck and Jens Bollerslev did not receive any specific grant from any funding agency in the public, commercial, or not-for-profit sector.

\section{References}

1 Giustina A, Casanueva FF, Cavagnini F, Chanson P, Clemmons D, Frohman LA, Gaillard R, Ho K, Jaquet P, Kleinberg DL, Pituitary Society \& the European Neuroendocrine Association et al. Diagnosis and treatment of acromegaly complications. Journal of Endocrinological Investigation 200326 1242-1247. (doi:10.1007/BF03349164)

2 Giustina A, Mazziotti G \& Canalis E. Growth hormone, insulin-like growth factors, and the skeleton. Endocrine Reviews 200829 535-559. (doi:10.1210/er.2007-0036)

3 Tamada D, Kitamura T, Onodera T, Tabuchi Y, Fukuhara A, Oshino S, Saitoh Y, Hamasaki T, Otsuki M \& Shimomura I. Rapid decline in bone turnover markers but not bone mineral density in acromegalic patients after transsphenoidal surgery. Endocrine Journal 201461 231-237. (doi:10.1507/endocrj.EJ13-0387) 
4 Ammann P \& Rizzoli R. Bone strength and its determinants. Osteoporosis International 200314 (Supplement 3) S13-S18.

5 Wassenaar MJ, Biermasz NR, Hamdy NA, Zillikens MC, van Meurs JB, Rivadeneira F, Hofman A, Uitterlinden AG, Stokkel MP, Roelfsema F et al. High prevalence of vertebral fractures despite normal bone mineral density in patients with long-term controlled acromegaly. European Journal of Endocrinology 2011164 475-483. (doi:10.1530/EJE10-1005)

6 Mazziotti G, Gola M, Bianchi A, Porcelli T, Giampietro A, Cimino V, Doga M, Gazzaruso C, De Marinis L \& Giustina A. Influence of diabetes mellitus on vertebral fractures in men with acromegaly. Endocrine 201140 102-108. (doi:10.1007/s12020-011-9486-x)

7 Claessen KM, Kroon HM, Pereira AM, Appelman-Dijkstra NM Verstegen MJ, Kloppenburg M, Hamdy NA \& Biermasz NR. Progression of vertebral fractures despite long-term biochemical control of acromegaly: a prospective follow-up study. Journal of Clinical Endocrinology \& Metabolism 201398 4808-4815. (doi:10.1210/ jc.2013-2695)

8 Seeman E, Wahner HW, Offord KP, Kumar R, Johnson WJ \& Riggs BL. Differential effects of endocrine dysfunction on the axial and the appendicular skeleton. Journal of Clinical Investigation 198269 1302-1309. (doi:10.1172/JCI110570)

9 Mazziotti G, Biagioli E, Maffezzoni F, Spinello M, Serra V, Maroldi R, Floriani I \& Giustina A. Bone turnover, bone mineral density, and fracture risk in acromegaly: a meta-analysis. Journal of Clinical Endocrinology \& Metabolism 2015100 384-394. (doi:10.1210/jc.20142937)

10 Madeira M, Neto LV, de Paula Paranhos Neto F, Barbosa Lima IC, Carvalho de Mendonca LM, Gadelha MR \& Fleiuss de Farias ML. Acromegaly has a negative influence on trabecular bone, but not on cortical bone, as assessed by high-resolution peripheral quantitative computed tomography. Journal of Clinical Endocrinology \& Metabolism 201398 1734-1741. (doi:10.1210/jc.2012-4073)

11 Ueland T, Fougner SL, Godang K, Schreiner T \& Bollerslev J. Serum GH and IGF-I are significant determinants of bone turnover but not bone mineral density in active acromegaly: a prospective study of more than 70 consecutive patients. European Journal of Endocrinology 2006155 709-715. (doi:10.1530/eje.1.02285)

12 Ueland T, Ebbesen EN, Thomsen JS, Mosekilde L, Brixen K, Flyvbjerg A \& Bollerslev J. Decreased trabecular bone biomechanical competence, apparent density, IGF-II and IGFBP-5 content in acromegaly. European Journal of Clinical Investigation 200232 122-128. (doi:10.1046/j.1365-2362.2002.00944.x)

13 Fogelman I \& Blake GM. Different approaches to bone densitometry. Journal of Nuclear Medicine 200041 2015-2025.

14 Hans D, Barthe N, Boutroy S, Pothuaud L, Winzenrieth R \& Krieg MA. Correlations between trabecular bone score, measured using anteroposterior dual-energy X-ray absorptiometry acquisition, and 3-dimensional parameters of bone microarchitecture: an experimental study on human cadaver vertebrae. Journal of Clinical Densitometry 201114 302-312. (doi:10.1016/j.jocd.2011.05.005)

15 Winzenrieth R, Michelet F \& Hans D. Three-dimensional (3D) microarchitecture correlations with $2 \mathrm{D}$ projection image gray-level variations assessed by trabecular bone score using high-resolution computed tomographic acquisitions: effects of resolution and noise. Journal of Clinical Densitometry 201316 287-296. (doi:10.1016/j. jocd.2012.05.001)

16 Pothuaud L, Benhamou CL, Porion P, Lespessailles E, Harba R \& Levitz P. Fractal dimension of trabecular bone projection texture is related to three-dimensional microarchitecture. Journal of Bone and Mineral Research 200015 691-699. (doi:10.1359/jbmr.2000.15.4.691)

17 Pothuaud L, Carceller P \& Hans D. Correlations between grey-level variations in $2 \mathrm{D}$ projection images (TBS) and $3 \mathrm{D}$ microarchitecture: applications in the study of human trabecular bone microarchitecture. Bone 200842 775-787. (doi:10.1016/j.bone.2007.11.018)
18 Kreider JM \& Goldstein SA. Trabecular bone mechanical properties in patients with fragility fractures. Clinical Orthopaedics and Related Research 2009467 1955-1963. (doi:10.1007/s11999-009-0751-8)

19 Hildebrand T, Laib A, Muller R, Dequeker J \& Ruegsegger P. Direct three-dimensional morphometric analysis of human cancellous bone: microstructural data from spine, femur, iliac crest, and calcaneus. Journal of Bone and Mineral Research 199914 1167-1174. (doi:10.1359/ jbmr.1999.14.7.1167)

20 Olarescu NC, Heck A, Godang K, Ueland T \& Bollerslev J. The metabolic risk in newly diagnosed patients with acromegaly is related to fat distribution and circulating adipokines and improves after treatment. Neuroendocrinology 2016103 197-206. (doi:10.1159/000371818)

21 Gjesdal CG, Aanderud SJ, Haga HJ, Brun JG \& Tell GS. Femoral and whole-body bone mineral density in middle-aged and older Norwegian men and women: suitability of the reference values. Osteoporosis International 200415 525-534. (doi:10.1007/s00198-0031573-2)

22 Godang K, Qvigstad E, Voldner N, Isaksen GA, Froslie KF, Notthellen J, Henriksen T \& Bollerslev J. Assessing body composition in healthy newborn infants: reliability of dual-energy x-ray absorptiometry. Journal of Clinical Densitometry 201013 151-160. (doi:10.1016/j.jocd.2010.01.121)

23 Godang K, Ueland T \& Bollerslev J. Decreased bone area, bone mineral content, formative markers, and increased bone resorptive markers in endogenous Cushing's syndrome. European Journal of Endocrinology 1999141 126-131. (doi:10.1530/eje.0.1410126)

24 Silva BC, Leslie WD, Resch H, Lamy O, Lesnyak O, Binkley N, McCloskey EV, Kanis JA \& Bilezikian JP. Trabecular bone score: a noninvasive analytical method based upon the DXA image. Journal of Bone and Mineral Research 201429 518-530. (doi:10.1002/jbmr.v29.3)

25 Cormier C, Lamy O \& Poriau S. TBS in routine clinical practice: proposals of use [internet]. (available from: http://www. medimapsgroup.com/upload/MEDIMAPS-UK-WEB.pdf. Plan-lesOutes, Switzerland: Medimaps Group; 2012, 2012.

26 Leib E, Winzenrieth R, Aubry-Rozier B \& Hans D. Vertebral microarchitecture and fragility fracture in men: a TBS study. Bone 201462 51-55. (doi:10.1016/j.bone.2013.12.015)

27 Bolanowski M, Daroszewski J, Medras M \& Zadrozna-Sliwka B. Bone mineral density and turnover in patients with acromegaly in relation to sex, disease activity, and gonadal function. Journal of Bone and Mineral Metabolism 200624 72-78. (doi:10.1007/s00774-005-0649-9)

28 Vestergaard P \& Mosekilde L. Fracture risk is decreased in acromegaly - a potential beneficial effect of growth hormone. Osteoporosis International 200415 155-159. (doi:10.1007/s00198-003-1531-z)

29 Ueland T, Bollerslev J, Hansen TB, Ebbesen EN, Mosekilde L, Brixen K, Flyvbjerg A \& Djoseland O. Increased cortical bone content of insulin-like growth factors in acromegalic patients. Journal of Clinical Endocrinology \& Metabolism 199984 123-127. (doi:10.1210/ jcem.84.1.5384)

30 Battista C, Chiodini I, Muscarella S, Guglielmi G, Mascia ML, Carnevale V \& Scillitani A. Spinal volumetric trabecular bone mass in acromegalic patients: a longitudinal study. Clinical Endocrinology 2009 70 378-382. (doi:10.1111/cen.2009.70.issue-3)

31 Bonadonna S, Mazziotti G, Nuzzo M, Bianchi A, Fusco A, De Marinis L \& Giustina A. Increased prevalence of radiological spinal deformities in active acromegaly: a cross-sectional study in postmenopausal women. Journal of Bone and Mineral Research 200520 1837-1844. (doi:10.1359/JBMR.050603)

32 Madeira M, Neto LV, Torres CH, de Mendonca LM, Gadelha MR $\&$ de Farias ML. Vertebral fracture assessment in acromegaly. Journal of Clinical Densitometry 201316 238-243. (doi:10.1016/j. jocd.2012.06.002)

33 Mazziotti G, Bianchi A, Bonadonna S, Cimino V, Patelli I, Fusco A, Pontecorvi A, De Marinis L \& Giustina A. Prevalence of vertebral 
fractures in men with acromegaly. Journal of Clinical Endocrinology \& Metabolism 200893 4649-4655. (doi:10.1210/jc.2008-0791)

34 Mazziotti G, Bianchi A, Porcelli T, Mormando M, Maffezzoni F, Cristiano A, Giampietro A, De Marinis L \& Giustina A. Vertebral fractures in patients with acromegaly: a 3-year prospective study. Journal of Clinical Endocrinology \& Metabolism 201398 3402-3410. (doi:10.1210/jc.2013-1460)

35 Hong AR, Kim JH, Kim SW, Kim SY \& Shin CS. Trabecular bone score as a skeletal fragility index in acromegaly patients. Osteoporosis International 201627 1123-1129. (doi:10.1007/s00198015-3344-2)

36 Kristo C, Jemtland R, Ueland T, Godang K \& Bollerslev J. Restoration of the coupling process and normalization of bone mass following successful treatment of endogenous Cushing's syndrome: a prospective, long-term study. European Journal of Endocrinology 2006 154 109-118. (doi:10.1530/eje.1.02067)

37 Bollerslev J, Jansson S, Mollerup CL, Nordenstrom J, Lundgren E, Torring O, Varhaug JE, Baranowski M, Aanderud S, Franco C et al. Medical observation, compared with parathyroidectomy, for asymptomatic primary hyperparathyroidism: a prospective, randomized trial. Journal of Clinical Endocrinology \& Metabolism 2007 92 1687-1692. (doi:10.1210/jc.2006-1836)

38 Lundstam K, Heck A, Mollerup C, Godang K, Baranowski M, Pernow Y, Varhaug JE, Hessman O, Rosen T, Nordenstrom J et al. Effects of parathyroidectomy versus observation on the development of vertebral fractures in mild primary hyperparathyroidism. Journal of Clinical Endocrinology \& Metabolism 2015100 1359-1367. (doi:10.1210/jc.2014-3441)

39 Ueland T. Bone metabolism in relation to alterations in systemic growth hormone. Growth Hormone \& IGF Research 200414 404-417. (doi:10.1016/j.ghir.2004.06.002)

40 Senn C, Gunther B, Popp AW, Perrelet R, Hans D \& Lippuner K. Comparative effects of teriparatide and ibandronate on spine bone mineral density (BMD) and microarchitecture (TBS) in postmenopausal women with osteoporosis: a 2-year open-label study. Osteoporosis International 201425 1945-1951. (doi:10.1007/s00198014-2703-8)

41 Olarescu NC \& Bollerslev J. The impact of adipose tissue on insulin resistance in acromegaly. Trends in Endocrinology and Metabolism 2016 27 226-237. (doi:10.1016/j.tem.2016.02.005)

Received 15 April 2016

Revised version received 18 May 2016

Accepted 23 May 2016 